\title{
Voilà, vous devez savoir « presque » tout sur le carcinome épidermoïde du canal anal...
}

\author{
J.-H. Lefevre \\ (C) Springer-Verlag France 2011
}

Son traitement repose donc en première intention sur la radiochimiothérapie 45 Gy avec un surdosage de 15 Gy pour les tumeurs ayant bien répondu après un délai d'attente de 4 semaines. La chirurgie reste néanmoins nécessaire pour environ $20 \%$ des patients (tumeur n'ayant pas répondu aux $45 \mathrm{~Gy}$, persistante après les $60 \mathrm{~Gy}$ ou en cas de récidive à distance). Sa morbidité périnéale a été réduite par les nouvelles techniques (lambeaux de grand droit, colostomie périnéale...).

Les questions qui restent en suspens restent cependant nombreuses :

- quid d'un traitement adjuvant après chirurgie ?
- Comment traiter les récidives postamputation?

- Quel va être l'impact de la vaccination HPV débutée, il y a quelques années?

- Comment réduire encore le taux d'amputation abdominopérinéale?

- Y a-t-il une alternative à l'amputation en cas d'échec du traitement médical...?

- Comment améliorer la qualité de vie après amputation?

Autant de questions qui seront, je l'espère, traitées dans le prochain dossier thématique sur le cancer épidermoïde $\mathrm{du}$ canal anal dans quelques années. 